\title{
Bilateral Unusual Course of the Median Nerve, Variation in Branching Pattern of the Brachial and Superficial Ulnar Arteries: A Rare Case Report of Multiple Neuroarterial Variation
}

\author{
Shahriar Ahmadpour ${ }^{1 \odot}$ Khadijeh Foghi $^{1}$ \\ ${ }^{1}$ Department of Anatomy, Faculty of Medicine, North Khorasan \\ University of Medical Sciences, Bojnurd, Iran \\ J Morphol Sci 2019;36:202-206.
}

\begin{abstract}
Address for correspondence Shahriar Ahmadpour, PhD, Department of Anatomy, Faculty of Medicine, North Khorasan University of Medical Sciences, Bojnurd, North Khorasan Province, Iran (e-mail: Shahahmadpour@gmail.com).
\end{abstract}

Abstract
Keywords
- anatomical variations
- median nerve
- ulnar artery
- ulnar collateral
arteries
- common
interosseous artery
- cadaver

Introduction Bilateral unusual course of the median nerve accompanied with variations of the brachial artery branching pattern are uncommon.

Materials and Methods During the routine educational dissection of an upper limb, an interesting neurovascular variation was found in a 45-year-old male cadaver.

Results We found a bilateral unusual and variant course of the median nerve in the arm region. The right median nerve, after formation, descended from the medial to the brachial artery, crossed the brachial artery anteriorly from medial to lateral, then inferiorly and lied medially to the distal third of the brachial artery, while the left median nerve ran medial to the brachial artery, passing anteriorly from medial to lateral, and, at the distal end of the arm, it buried itself in the brachialis muscle. Another set of findings were absence of the superior and inferior ulnar collateral arteries, superficial ulnar artery in the forearm and common interosseus artery originated from radial artery.

Conclusion These types of compound neurovascular variations are of great importance in orthopedic, vascular, reconstructive surgeries and even in routine nursing care.

\section{Introduction}

The median nerve is formed by the fusion of the lateral and medial roots coming from the lateral and medial cords of the brachial plexus, respectively. ${ }^{1}$ After this fusion, the median nerve descends anterior to the axillary artery and the upper third of the brachial artery. In the middle of arm, the median nerve crosses from the lateral to the medial side of the brachial artery. ${ }^{2,3}$ Anatomical variations of the median nerve have been described by many authors. ${ }^{4,5}$ Budhiraja et al (2011) reported the existence of an additional root in the formation of the median nerve in $26.4 \%$ of upper limbs, (10) Shahriar Ahmadpour's ORCID is https://orcid.org/0000-0002-7744-
3358 .

received

September 5, 2018

accepted

March 28, 2019

10.1055/s-0039-1691755. ISSN 2177-0298. unusual low formation of the median nerve in the arm in front of the brachial artery in $18.4 \%$ of upper limbs, and median nerve formation medial to the axillary artery in $10.3 \%$ of upper limbs. ${ }^{6}$ Mat Tiab et al (2017) studied the distribution of variations of the median nerve. According to their study, normal formation of the median nerve from two roots represented $63.6 \%$ (14) and $72.7 \%$ (16) for the left and right upper limbs, respectively. ${ }^{7}$ Three other variations of median nerve formation that have been observed are: one root, three roots, and four roots. Three roots forming the median nerve were found in $\sim 36.4 \%(8)$ in the left upper limb, and $18.2 \%$ (4) in the right one. More variations were observed in the right upper limb. Median nerves formed from one root and four roots were observed in $4.5 \%$ of upper limbs, each. A normal course of the median nerve in relation

Copyright @ 2019 by Thieme Revinter

Publicações Ltda, Rio de Janeiro, Brazil

License terms

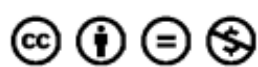


to the axillary artery had the same distribution on the left and right sides, $81.8 \%$ (18). In $13.6 \%$ (3) of the samples, the median nerve was running posterior to the axillary artery, and in $4.5 \%$ (1) of the cases, it ran medial to the axillary artery in the left upper limb. Beheiry ${ }^{8}$ also reported anatomical variations of the median nerve distributions and communication in the arm region. According to Beheiry's report, in $1.7 \%$ of the cases, the median nerve gave off muscular branches to the brachialis muscle. Variations in the vascular pattern of the upper limb have been also frequently observed. According to the Compendium of Human Anatomic Variation, major variations are present in the brachial artery of $\sim 25 \%$ of the subjects studied. The variations in the form of high proximal division into terminal branches occur in the radial artery (15\%), ulnar artery (2\%), and common interosseous artery (very rarely). ${ }^{9}$ According to the studies, at least four major types of variation of the brachial artery have been described, with trifurcation of the brachial artery, double profunda brachial artery (DPBA), high division of brachial artery (HDBA) and high origin of radial artery (HORA) being the most commonly observed variations. ${ }^{10}$ The variations described in the following case are quite different and rare in terms of bilateral neurovascular variations. We observed a bilateral variant configuration of the median nerve accompanied by variation of brachial artery branching in the arm region and superficial ulnar artery in a male cadaver. Multiple neurovascular variations in the upper extremities are of potential clinical implications, as this is a frequent site of injury that is also involved in many surgical and invasive procedures. Therefore, knowledge of such multiple variations could help to avoid malpractice.

\section{Case Report}

During a routine educational dissection, a bilateral variation of the brachial artery was observed in a 45-year-old male cadaver. We found a bilateral, unusual and variant course of the median nerve in the arm region. The right median nerve, after its formation, descended from the medial to the brachial artery, crossed the brachial artery anteriorly from medial to lateral, then inferiorly and lied medially to the distal third of the brachial artery (-Fig. 1). The left median nerve showed a different course to the brachial artery. It ran medial to the brachial artery, passing anteriorly from medial to lateral, and, at the distal end of the arm, it buried itself in the brachialis muscle (-Figs. 2, 3). Additionally, a small anomalous branch was observed arising from the lateral aspect of the left median nerve passed inferiorly into the left cubital fossa (-Fig. 2). Further inspection revealed that the right superior and inferior ulnar collateral arteries were replaced by two arteries arising from the lateral side of the brachial artery. The right superior one ran deep into brachialis muscle as a muscular branch, while the right lower (inferior) one descended between the biceps and brachialis muscles (-Fig. 4). The left superior and inferior ulnar collateral arteries were also replaced by one muscular branch aroused from the lateral side of the left brachial artery. Further examination in the

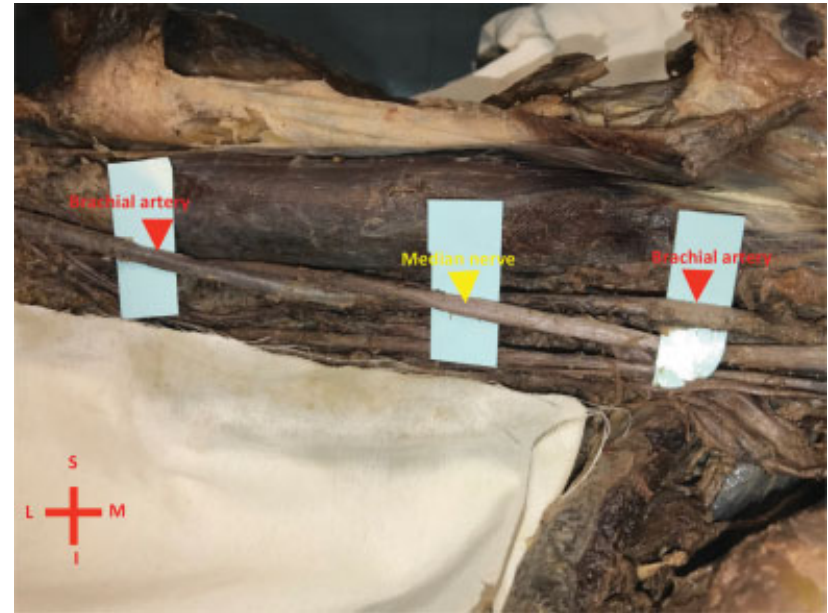

Fig. 1 Right arm. The right median nerve, after formation in the medial side of the brachial artery, crosses it anteriorly and then runs inferior to the brachial artery and lies medial to it.

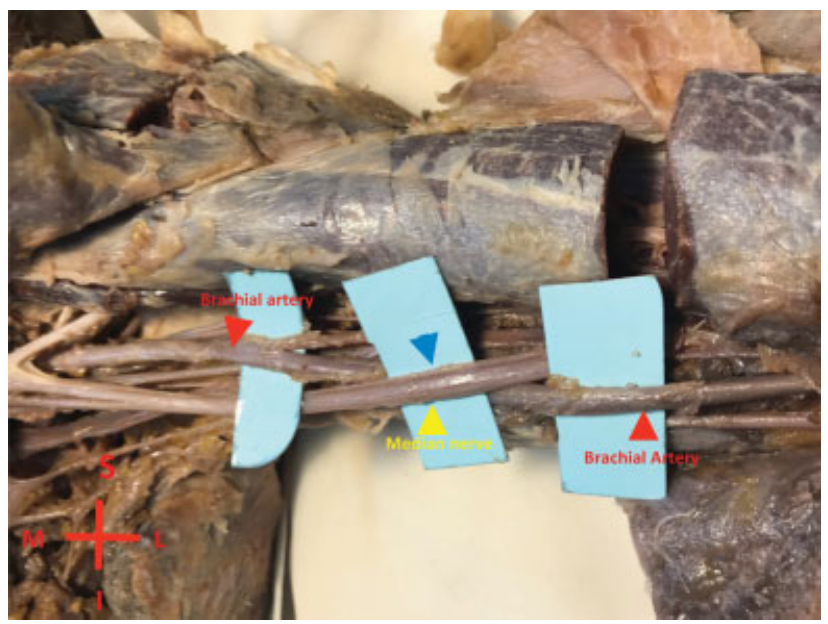

Fig. 2 The left median nerve with a thin, small anomalous branch (blue arrowhead) formed on the medial side of the brachial artery (red arrowhead) descending from its medial to lateral side.

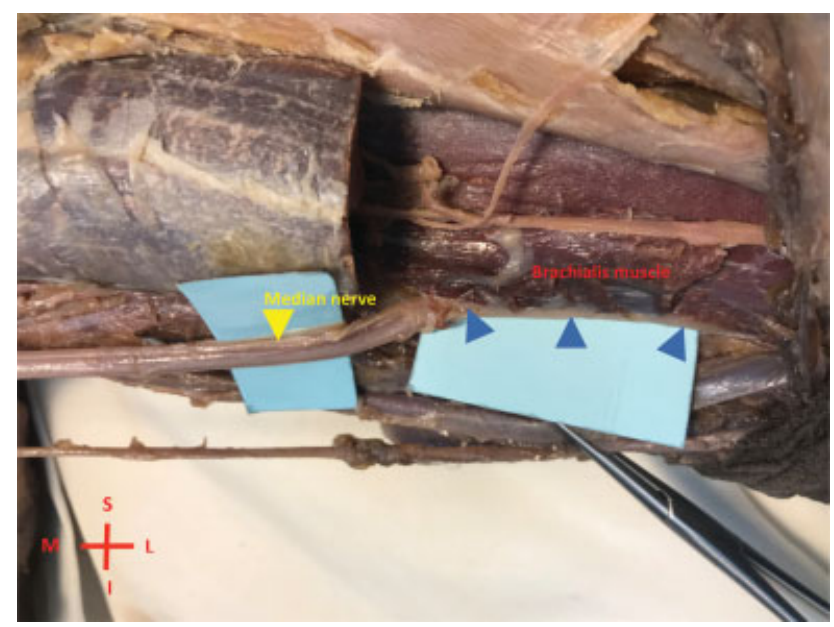

Fig. 3 Photograph shows the distal third of the left median nerve buried into the brachialis (blue arrowheads). 


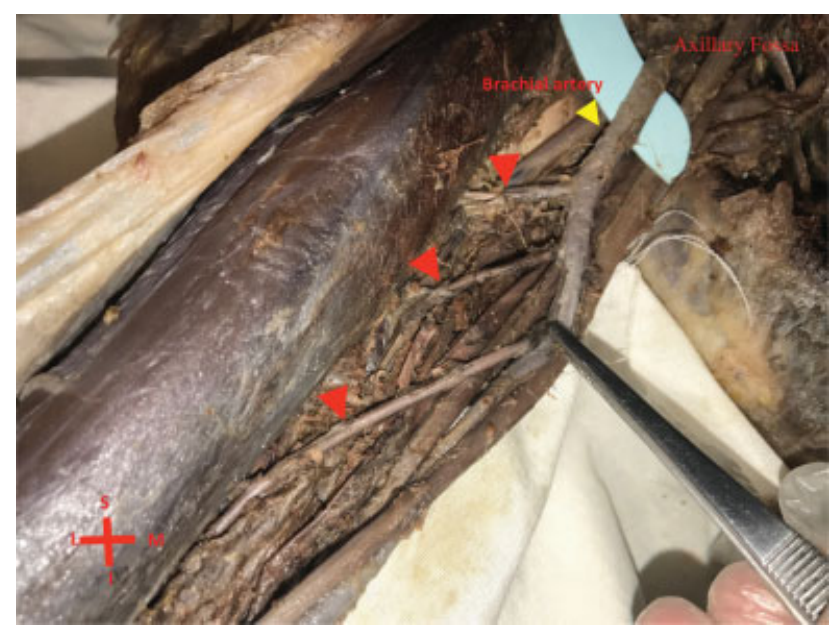

Fig. 4 Right arm and right brachial artery (yellow arrowhead). Right superior and inferior ulnar collateral arteries are replaced by two arteries arising from the lateral side of the brachial artery

cubital region revealed that the right and left common interosseus arteries aroused from the radial and ulnar arteries were superficial to the flexor carpi radialis (both sides), and the ulnar recurrent arteries were absent bilaterally. (-Figs. 5, 6)

\section{Discussion}

According to the standard anatomy text books, the median nerve is formed by a fusion of two roots, and, after joining, it crosses the brachial artery from the lateral to the medial side. However, variations of the median nerve, including abnormal communication with other nerves, splitting of the median nerve, and formation of the median nerve by more than two roots, have been reported. ${ }^{11-13}$ In the present case, a bilateral unusual course of the median nerve in the arm region accompanied with brachial artery branching pattern variations was found. Unusual course of the median nerve has been reported earlier by a few authors. Singh et al reported that the median nerve was formed medial to the

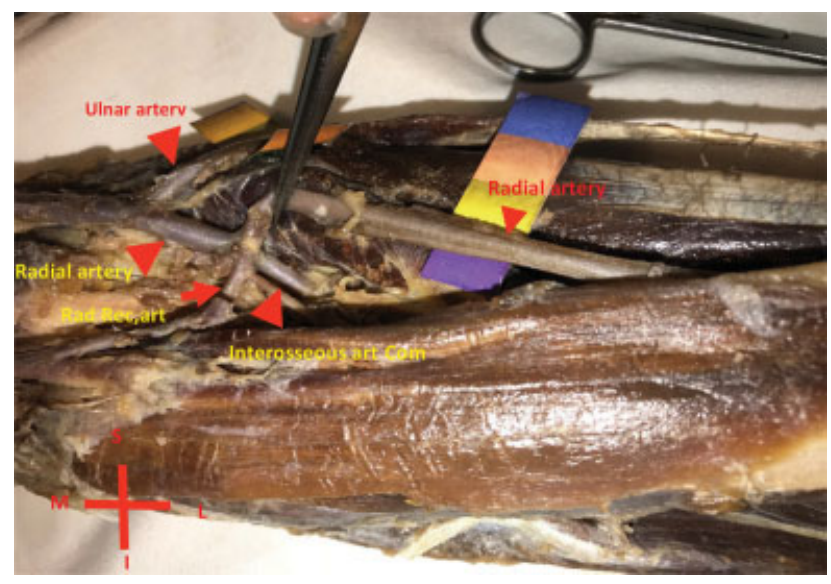

Fig. 5 Left cubital region. Common interosseous artery arising from the radial artery. A single recurrent branch (radial recurrent artery [rad rec art]) stems from the radial artery.

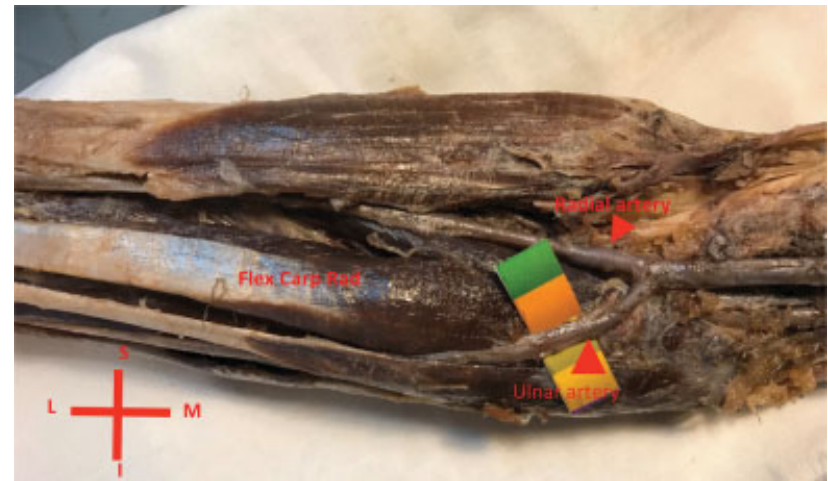

Fig. 6 The ulnar artery passing superficially to the flexor carpi radialis (Flex Carp Rad).

brachial artery and crossed it anteriorly from the medial to the lateral side. ${ }^{1}$ There are also reports in which the median nerve was formed medial to the axillar artery. ${ }^{14-16}$ In another study, median nerve formation medial to the axillar artery was reported in $6.12 \%$ of upper limbs. ${ }^{6}$ Another study reported median nerve descending medial to the brachial artery in $6 \%$ of cases. ${ }^{17}$ Chitra also reported median nerve coursing medial to the brachial artery. ${ }^{14}$ In the present case, we observed a rare bilateral variation of the median nerve course in the arm region. On the right side, the median nerve double crossed the brachial artery. Precisely, the right median nerve descended medial to the brachial artery in the upper third of the arm; then, it crossed the brachial artery anteriorly from medial to lateral; in the middle third of the arm, it hooked the brachial artery, and, in the distal third, it crossed from lateral to medial inferiorly. The left medial nerve descended medial to the brachial artery in the upper half of the arm, crossing the artery anteriorly at the junction of the upper and lower halves of the arm. In the distal third of the arm, the left median nerve ran lateral to the brachial artery, where it buried itself deeply into the brachial muscle. There are comprehensive studies have dealt with the variations of the median nerve. For instance, Buhiraja et $\mathrm{al}^{6}$ have studied 174 upper limbs and reported the variations of median nerve formation in terms of roots and course in relation to the brachial artery. To the best of our knowledge, the variation presented here of median nerve variation has not been reported. Helical relation of the right median nerve to the brachial artery, and the deeply buried left median nerve with a different course to the right median nerve may lead to neurologic signs in the upper limbs, which would not be diagnosed by routine physical examination. Peripheral nerve injury in the upper extremity is common, and certain peripheral nerves are at an increased risk of injury because of their anatomic location. Risk factors include a superficial position, a long course through an area at high risk of trauma, and a narrow path through a bony canal. The most common nerve entrapment injury is carpal tunnel syndrome, which has an estimated prevalence of $3 \%$ in the general population, and 5 to $15 \%$ in the industrial setting. ${ }^{18}$ Spinner et al $^{19}$ escribed entrapment of the median nerve caused by compression in the distal arm because of an accessory. It was characterized by the paresis 
or paralysis of muscles innervated by the anterior interosseous branch of the median nerve-the flexor pollicis longus, the flexor digitorum profundus, and the pronator quadratus -as well as other more proximal median nerve-innervated muscles, namely, the pronator teres and flexor carpi radialis. The case we presented here raised another possible cause of median nerve entrapment in vivid anatomy, which has not been noticed in clinical literatures. Another set of findings were bilateral variations of brachial artery branching pattern. We observed bilateral vascular variations that have not been reported before. For instance, the right superior and inferior ulnar collateral arteries were absent; instead, two branches aroused from the lateral side of the brachial artery with muscular distribution. The left superior and inferior ulnar collateral arteries were replaced with a single branch arising from the lateral side of the brachial artery. In the cubital region, the ulnar artery in both sides ran superficially, while the common interosseus artery aroused from the radial artery. To date, numerous anatomical variations in the brachial artery and its branching pattern have been reported. The most commonly reported variation in upper limb arteries is the higher division of brachial artery into the radial and ulnar arteries above the cubital fossa. For instance, Kaur et $\mathrm{al}^{20}$ studied 40 upper limbs and observed a higher division of the brachial artery into radial and ulnar arteries above the elbow joint in 4 cases, trifurcated ulnar artery at the lower border of pronator teres in 3 cases, and inferior ulnar collateral artery absence in 4 cases. In another study, Chakravarthi et al $^{21}$ evaluated 140 upper limb specimens and reported accessory brachial artery as the main arterial variation limb. Superficial ulnar artery is an uncommon variation in which the ulnar artery is having its course superficial to the flexors of the forearm and may arise directly from the axillary or brachial arteries. The presence of a superficial ulnar artery seems to be a rare variation, with an incidence of 0.7 to $7 \%$ and has unilateral preponderance. $^{22}$ Senanayake et $\mathrm{al}^{23}$ reported superficial ulnar artery aroused from the upper one third of the brachial artery. Quadros et $\mathrm{al}^{22}$ also reported a unilateral superficial ulnar artery and a common interosseous stemming from the radial artery. The most frequently reported finding pertinent to the present case is the variation described by Quadros, but we found bilateral superficial ulnar artery and common interosseous as the principle branch of the ulnar artery, stemming from the radial artery. Bilateral multiple neuroarterial variations in the upper extremities are considered challenging in surgical procedures. The case presented here showed a series of neurovascular variations, including bilateral unusual course of the median nerve and absent superior and inferior ulnar collateral arteries. The observed variations in the arterial pattern of the brachial artery and the median nerve assume significance during clinical examinations of neurologic symptoms, vascular surgeries and local anesthesia procedures. Unusual course of the peripheral nerves may lead to nerve entrapments and subsequent muscles paresis. Additionally, absence of major arterial branches-namely ulnar collaterals around the elbow joint-could potentially trigger neurologic symptoms.
Therefore, the importance of such multiple variations goes beyond the merely anatomical variation reports and need to be considered and emphasized in clinical practice. Some of the most frequent types of arterial variations, such as superficial ulnar artery, can be mistaken as veins during venipuncture.

\section{Conclusion}

Regarding the complex variations presently described, we believe that knowledge of such compound unusual anatomical relations and patterns is not only of great importance in orthopedic and vascular surgeries, but it should also be considered in common practices.

Conflicts of Interest

The authors have no conflicts of interest to report.

\section{References}

1 Singh R, Wadhwan M. Unusual course of median nerve in arm and forearm. Int J Anat Var 2016;9:82-84

2 Haviarova Z, el Falougy HA, Killingerova A. Atypical course of the median nerve. Bratisl Lek Listy 2001;102(08):372-373

3 Singh S, Rani N, Kaushal P, Kumar H, Sharrif A, Roy TS. Anomalous cutaneous branch of median nerve in arm: a report of anatomical variation with clinical implications. Anat Cell Biol 2014;47(02): $138-140$

4 Saralaya V, Nayak RS, Sequeira S, Madhyastha S, Krishnamurthy A, D'Costa S. An unusual tunnel formation in the arm and its clinical significance. Int J Anat Var 2009;2:27-28

5 Vollala VR, Raghunathan D, Rodrigues V. Nerve compressions in upper limb: a case report. Neuroanat 2005;4:35-36

6 Budhiraja V, Rastogi R, Asthanan AK. Anatomical variation of median nerve formation: embryological and clinical correlation. Journal of Morphological Sciences 2011;28:283-286

7 Mat Taib CN, Hassan SNA, Esa N, Mohd Moklas MA, San AA. Anatomical variations of median nerve formation, distribution and possible communication with other nerves in preserved human cadavers. Folia Morphol (Warsz) 2017;76(01):38-43

8 Beheiry EE. Anatomical variations of the median nerve distribution and communication in the arm. Folia Morphol (Warsz) 2004; 63(03):313-318

9 Bergman RA, Thompson SA, Afifi AK, Saadeh FA. Compendium of human anatomic variation. Baltimore: Urban \&Schwarzenberg; 1988

10 SHIVANAL U, GOWDA TMS. A study of variations in brachial artery and its branching pattern. International Journal of Research in Medical Sciences 2015;3(06):1392-1396

11 Eglseder WA Jr, Goldman M. Anatomic variations of the musculocutaneous nerve in the arm. Am J Orthop 1997;26(11):777-780

12 Chauhan R, Roy TS. Communication between the median and musculocutaneous nerve- a case report. J Anat Soc India 2002; 51:72-75

13 George BM, Nayak SM. Median nerve and brachial artery entrapment in the abnormal brachialis muscle- a case report. Neuroanat 2008;7:41-42

14 Chitra R. Multiple bilateral neuroanatomical variations of the nerves of the arm. Neuroanat 2007;6:43-45

15 Singhal S, Rao VV, Ravindranath R. Variations in brachial plexus and the relationship of median nerve with the axillary artery: a case report. J Brachial Plex Peripher Nerve Inj 2007;2(21):21

16 Satyanarayana N, Vishwakarma N, Kumar GP, Guha R, Dattal AK, Sunitha P. Rare variations in the formation of median nerveembryological basis and clinical significance. Nepal Med Coll J 2009;11(04):287-290 
17 Channa B, Patil S, Shinde VK, Jevoor PS, Nidoni M. A study of anatomical variations of median nerve in human cadavers. Int J Biomed Res 2013;4(12):682-690

18 Atroshi I, Gummesson C, Johnsson R, Ornstein E, Ranstam J, Rosén I. Prevalence of carpal tunnel syndrome in a general population. JAMA 1999;282(02):153-158

19 Spinner RJ, Carmichael SW, Spinner M. Partial median nerve entrapment in the distal arm because of an accessory bicipital aponeurosis. J Hand Surg Am 1991;16(02):236-244

20 Kaur A, Sharma A, Shram M. Variation in Branching Pattern of Brachial Artery. Int J Sci Stud 2017;5(01):213-217
21 Chakravarthi KK, Ks S, Venumadhav N, Sharma A, Kumar N. Anatomical variations of brachial artery - its morphology, embryogenesis and clinical implications. J Clin Diagn Res 2014;8(12): AC17-AC20

22 Quadros LS, Bhat N, D’Souza AS. Superficial Ulnar Artery: A Case Report of its Unusual Course. Malays J Med Sci 2015;22(03): 65-67

23 Senanayake KJ, Salgado S, Rathnayake MJ, Fernando R, Somarathne K. A rare variant of the superficial ulnar artery, and its clinical implications: a case report. J Med Case Reports 2007; $1: 128$ 